\title{
Article \\ EEG Frontal Asymmetry in Dysthymia, Major Depressive Disorder and Euthymic Bipolar Disorder
}

\author{
Chiara Spironelli ${ }^{1,2, * \mathbb{C}}$, Francesca Fusina ${ }^{1,2}$, Marco Bortolomasi $^{3}$ and Alessandro Angrilli ${ }^{1,2}$ \\ 1 Department of General Psychology, University of Padova, 35131 Padova, Italy; \\ francesca.fusina@phd.unipd.it (F.F.); alessandro.angrilli@unipd.it (A.A.) \\ 2 PNC_-Padova Neuroscience Center, University of Padova, 35131 Padova, Italy \\ 3 Villa S. Chiara Health Clinic-Quinto di Verona, 37142 Verona, Italy; marcobortolomasi.vr@gmail.com \\ * Correspondence: chiara.spironelli@unipd.it; Tel.: +39-049-827-6619
}

Citation: Spironelli, C.; Fusina, F.; Bortolomasi, M.; Angrilli, A. EEG Frontal Asymmetry in Dysthymia, Major Depressive Disorder and Euthymic Bipolar Disorder. Symmetry 2021, 13, 2414. https://doi.org/ 10.3390/sym13122414

Academic Editor: Vilfredo De Pascalis

Received: 21 September 2021

Accepted: 9 December 2021

Published: 13 December 2021

Publisher's Note: MDPI stays neutral with regard to jurisdictional claims in published maps and institutional affiliations.

\begin{abstract}
In the last few decades, the incidence of mood disorders skyrocketed worldwide and has brought an increasing human and economic burden. Depending on the main symptoms and their evolution across time, they can be classified in several clinical subgroups. A few psychobiological indices have been extensively investigated as promising markers of mood disorders. Among these, frontal asymmetry measured at rest with quantitative EEG has represented the main available marker in recent years. Only a few studies so far attempted to distinguish the features and differences among diagnostic types of mood disorders by using this index. The present study measured frontal EEG asymmetry during a 5-min resting state in three samples of patients with bipolar disorder in a Euthymic phase (EBD, $n=17$ ), major depressive disorder (MDD, $n=25$ ) and persistent depressive disorder (PDD, $n=21$ ), once termed dysthymia. We aimed to test the hypothesis that MDD and PDD lack the typical leftward asymmetry exhibited by normal as well as EBD patients, and that PDD shows greater clinical and neurophysiological impairments than MDD. Clinical scales revealed no symptoms in EBD, and significant larger anxiety and depression scores in PDD than in MDD patients. Relative beta (i.e., beta/alpha ratio) EEG asymmetry was measured from lateral frontal sites and results revealed the typical greater left than right frontal beta activity in EBD, as well as a lack of asymmetry in both MDD and PDD. The last two groups also had lower bilateral frontal beta activity in comparison with the EBD group. Results concerning group differences were interpreted by taking into account both the clinical and the neurophysiological domains.
\end{abstract}

Keywords: frontal asymmetry; EEG; major depressive disorder; persistent depressive disorder; dysthymia; euthymic bipolar disorder

\section{Introduction}

In recent years, together with anxiety, mood disorders have represented the most prevalent and heterogeneous mental diseases worldwide. A possible research approach for such a complex condition may rely on the severity of mood symptoms. In the present study, together with major depressive disorder (MDD) patients, we also recruited persistent depressive disorder (PDD, traditionally termed dysthymia in previous versions of the DSM) [1] patients. PDD is a chronic condition marked by depressed mood for most of the day, almost every day, for at least two years [1], and it is closely associated with MDD [2-4], although some authors suggest that the former is less severe than the latter [3]. However, the persistence of depressive mood symptoms may induce important effects in patients' quality of life, as suggested by their high comorbidity rates with anxiety, substance addiction and personality disorders [4]. In their recent review, Schramm and colleagues [5] quoted only two experimental studies focused on the psychobiological neuroimaging of PDD. Ravindran and colleagues [6] suggested a core role of dorsolateral prefrontal cortex (DLPFC) hypoactivation in mood disorders: while lower DLPFC activity in the left hemisphere represented a shared substrate common to depressive disorders, right 
hypoactivation was associated with positive emotional processing and, compared to controls, characterized PDD patients' decreased attention towards positive stimuli, a probable marker of their anhedonia. Vilgis and colleagues [7] acquired fMRI data while young PDD patients and healthy controls carried out a mental rotation task. Results revealed decreased activation in PDD patients' frontal regions, including the left ventrolateral and dorsolateral prefrontal cortices, the dorsomedial prefrontal cortex, the anterior cingulate cortex and the frontal pole. In addition to this limited literature, during an emotional task [8] a lifetime MDD patient group exhibited lower frontal activity than participants without current/past MDD or dysthymia in all conditions. Altered frontal EEG asymmetry was also found in patients with a history of depressive disorders, who exhibited lower relative left frontal activity than never-depressed individuals.

Past research on MDD is wider when compared with that carried out on PDD patients, both considering active task execution and during resting state conditions. Most results from studies carried out on MDD revealed that hypofrontality may represent a reliable biological marker for these patients. Indeed, compared with healthy controls, MDD patients' hypofrontality (especially in the left hemisphere) has been found at rest both with neuroimaging and electrophysiological techniques, especially when analyzing slow electroencephalographic (EEG) activity [9-11]. Indeed, one of the most investigated psychophysiological markers of depressive symptoms is frontal asymmetry measured at rest [9,12-14]. In particular, greater left frontal activity has been associated with behavioral approach activation, goal attainment and manic symptoms, whereas decreased left frontal activation was associated with withdrawal activation, failure, loss, anxious, and depressive symptoms [15]. Most EEG studies showed greater alpha rhythm at left vs. right sites in MDD patients compared with healthy controls, regardless of closed/open eye conditions $[9,16-18]$. On the other hand, some studies revealed no direct association between left frontal hypoactivity and MDD, probably due to methodological differences in data collection as well as to patients' heterogeneity $[19,20]$. The review by Jesulola and colleagues [21] systematically analyzed frontal alpha asymmetry literature focusing on the inconsistency of results on frontal lobe asymmetry in MDD patients. Koek and colleagues [22] followed 13 bipolar patients for two years, and, using multiple measures across time, found that during their manic phases there was a leftward EEG frontal activation, whereas during their depressive episodes there was a rightward EEG frontal activation. In line with these findings, a meta-analysis on resting state alpha asymmetry as a marker of MDD found an overall null effect size [14], suggesting that the large heterogeneity in frontal asymmetry observed across studies is probably due to a number of critical variables that were not analyzed (e.g., putting together different phenotypical subtypes, gender, age). One relevant source of heterogeneity might be represented by the mixing of MDD and PDD patients within clinical samples enrolled in the studies. Indeed, only recently dysthymia, renamed in PDD, has been more clearly differentiated from MDD. These results clearly challenge the idea that frontal asymmetry alone represents a simple, straightforward diagnostic marker of depression. Additional measures based on either the selection of clinical samples considering the main symptoms (e.g., with and without anxiety comorbidity) or the use of other EEG bands, methods or new paradigms could help reduce this inconsistency. Looking at the paradigm, it would be important to explore frontal asymmetry as a marker during specific tasks rather than leaving participants free in resting state. Concerning the methods, more sophisticated mathematical approaches such as the SGWT (Spectral Graph Wavelet Transform [23]), the FD-NG (Fractal Dimension of the Undirected Graph [24]) and the WSN (Wireless Sense Network [25]) might represent innovative methods with respect to the classical EEG approach. Finally, EEG bands other than the alpha rhythm have been less investigated, but they could reveal greater reliability as potential markers of mood disorders. Indeed, the beta band has been used in our recent study [13] as a possible marker of patients' altered frontal activity at rest and during a linguistic task execution. Compared with healthy adults, MDD patients showed a lack of frontal asymmetry and a significantly lower activation of left frontal sites. Furthermore, positive affect in MDD 
patients was positively correlated with left frontal EEG beta amplitude, suggesting that higher beta EEG activity on left frontal sites accounts for an optimistic and positive attitude. Therefore, the beta band appears to be a promising EEG index in the study of frontal asymmetries. Nevertheless, this rhythm is usually associated with task execution, rather than a resting state condition, that is in turn typically linked to alpha modulations. To mediate this issue, the combination of alpha and beta EEG bands (beta/alpha ratio) takes into account both the inhibitory component, measured by alpha EEG, and the activation component, represented by the beta, merged into one measure, with the further advantage of statistically normalizing these measures across participants (i.e., the ratio between the two measures within subjects). Indeed, in a past experiment co-measuring PET and EEG in a healthy sample at rest, greater global and voxel-wise positive correlation was found between high frequency EEG bands (namely high-beta and gamma) and brain metabolism, whereas the alpha band showed the greatest negative correlation [26].

Currently, there is no clear evidence on whether PDD and MDD differ at a neurophysiological level, and how the three mood disorders (bipolar disorder, major depression, and dysthymia) differ in a direct comparison of their frontal asymmetry. Looking at the severity of depressive symptoms, PDD seems to be characterized by more subtle impairments compared to MDD. Also considering other clinical observations, PDD patients are expected to have more steadily altered neural circuits, due to the long persistence of their disorder. Therefore, in the present study we expected PDD patients to display greater alterations compared to MDD. To this end, in addition to MDD, we recruited a group of PDD patients and a sample of non-remitting bipolar outpatients in a euthymic state. All euthymic bipolar disorder (EBD) outpatients showed minimum residual mood symptoms with no current psychotic symptoms, satisfying a (sub)clinical condition referred to as euthymia. It is necessary to highlight that, when experimental designs are applied to the study of psychiatric samples, pharmacological treatment may account for important effects, thus representing a confounding variable that interacts with other experimental factors manipulated by researchers (e.g., [27,28]). Some drugs alter cognitive functioning, and the long-lasting treatments typical of chronic disorders contribute to a progressive worsening of patients' performances with respect to healthy adult samples [27]. In this perspective, euthymic patients could represent probably the best control group in clinical studies, as they show no active signs of disease but, at the same time, they share with depressed or bipolar patients (at least part of) a temporal continuity of pharmacological treatment. As a result of this, past research showed asymmetry changing in bipolar patients depending on their mood phase [22], so we expected EBD patients to show normal leftward EEG frontal asymmetry. Thus, the goals of the present study followed two main goals: first, we aimed at investigating whether EEG frontal asymmetry may represent a biomarker differentiating MDD and PDD, and whether it could support the hypothesis that PDD is a more severe disorder characterized by higher levels of anxiety, lower positive mood and higher pessimistic attitude. Secondly, we aimed at studying EEG frontal beta/alpha ratio, and its possible correlations with mood symptom severity and affective state measured by means of the Positive and Negative Affect Schedule (PANAS [29]). According with Spironelli and colleagues [13], we expected lower left frontal activity (beta/alpha ratio) in PDD than MDD, but greater left frontal ratio in EBD patients serving as clinically matched controls.

\section{Results}

Table 1 shows the results of the analyses carried out on socio-demographical and clinical data.

Groups had similar age, handedness and gender distributions, but revealed different levels of education [30] $\left(F_{2,60}=7.14, p=0.001\right)$ : EBD participants had higher educational levels than the other clinical samples (all $p s<0.01$ ). For this reason, education was used as a covariate factor in the analysis of both clinical scales and electrophysiological data.

Considering the severity of mood symptoms, the group factor was significant in all the scales we administered, with PDD patients showing the more severe clinical picture overall (Figure 1). 
Table 1. Socio-demographical and clinical data of the three patient groups (mean \pm Standard Errors).

\begin{tabular}{cccccc}
\hline & $\begin{array}{c}\text { EBD } \\
(\boldsymbol{n}=\mathbf{1 7})\end{array}$ & $\begin{array}{c}\text { MMD } \\
(\boldsymbol{n}=\mathbf{2 5})\end{array}$ & $\begin{array}{c}\text { PDD } \\
(\boldsymbol{n}=\mathbf{2 1})\end{array}$ & \multicolumn{2}{c}{ Statistics } \\
\hline Socio-demographical data & & & & & \\
\hline Age (years) & $51.5 \pm 2.76$ & $53.8 \pm 2.28$ & $59.1 \pm 2.48$ & $F_{2,60}=2.31$ & $n s$ \\
Gender & $7 \mathrm{M}-10 \mathrm{~F}$ & $5 \mathrm{M}-20 \mathrm{~F}$ & $7 \mathrm{M}-14 \mathrm{~F}$ & all $\chi^{2}{ }_{1} \leq 2.22$ & $n s$ \\
Education (years) & $14.8 \pm 0.90$ & $10.6 \pm 0.74$ & $11.1 \pm 0.81$ & $F_{2,60}=7.14$ & $p=0.001$ \\
Handedness & $92.5 \pm 1.87$ & $94.0 \pm 1.54$ & $98.0 \pm 1.68$ & $F_{2,60}=2.67$ & $n s$ \\
\hline Clinical data & & & & & \\
\hline HAM-D & $3.0 \pm 2.14$ & $8.1 \pm 1.67$ & $15.0 \pm 1.79$ & $F_{2,59}=37.90$ & $p<0.001$ \\
STAI-Y1 & $35.3 \pm 5.94$ & $30.9 \pm 4.64$ & $42.2 \pm 4.98$ & $F_{2,59}=5.79$ & $p<0.01$ \\
PANAS-PA & $28.2 \pm 4.13$ & $30.9 \pm 3.12$ & $24.0 \pm 3.35$ & $F_{2,59}=4.66$ & $p=0.01$ \\
PANAS-NA & $18.5 \pm 3.99$ & $19.2 \pm 3.02$ & $30.0 \pm 3.24$ & $F_{2,59}=15.27$ & $p<0.001$ \\
\hline
\end{tabular}

Note: EBD, euthymic bipolar disorder (EBD); MDD, major depressive disorder; PDD, persistent depressive disorder; HAM-D, Hamilton Rating Scale for Depression; STAI-Y1, State-Trait Anxiety Inventory-state form; PANAS-PA, Positive and Negative Affect Schedule-Positive Affect; PANAS-NA, Positive and Negative Affect Schedule-Negative Affect.
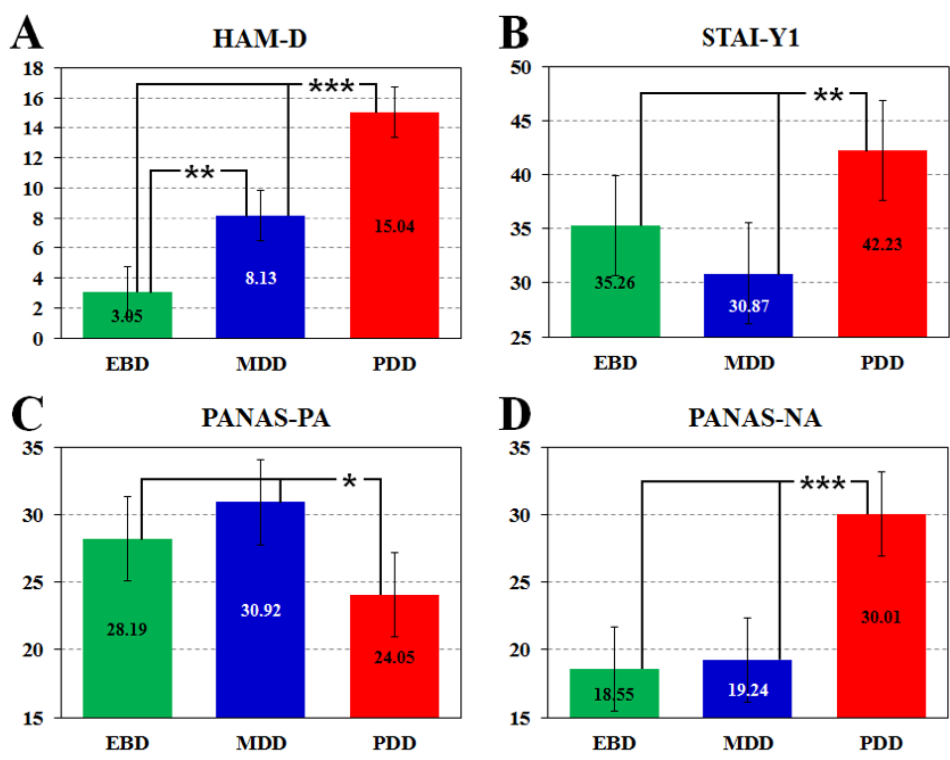

Figure 1. Severity of mood symptoms analyses: significant group main effect on (A) Hamilton Rating Scale for Depression (HAM-D), (B) State-Trait Anxiety Inventory-state form (STAI-Y1), (C) Positive Affect (PA) and (D) Negative Affect (NA) of the Positive and Negative Affect Schedule (PANAS). Asterisks: Significant post hoc comparisons (Newman-Keuls, $p<0.05)$. Note: EBD, euthymic bipolar disorder (EBD); MDD, major depressive disorder; PDD, persistent depressive disorder; ${ }^{*} p<0.05$; ** $p<0.01 ;{ }^{* * *} p<0.001$.

In detail, PDD patients reached the highest scores in the Hamilton Rating Scale for Depression (HAM-D [31]) $\left(F_{2,59}=37.90, p<0.001\right)$ with respect to all other groups (all ps $<0.001)$, whereas MDD patients showed higher scores than EBD participants $(p<0.01$; Figure 1A). PPD patients were also more anxious than both MDD and EBD individuals (all ps < 0.01), as revealed by the group effect at the State-Trait Anxiety Inventory-state form (STAI-Y1 [32]) $\left(F_{2,59}=5.79, p<0.01\right.$; Figure 1B). Furthermore, PDD patients exhibited the lowest Positive Affect (PANAS-PA) (all $p \mathrm{~s}<0.05)$ and the highest Negative Affect (PANAS-NA) (all $p$ s $<0.001)$ scores at the PANAS [29] subscales $\left(F_{2,59}=4.66, p=0.01\right.$, Figure $1 C$, and $F_{2,59}=15.27, p<0.001$, Figure 1D) when compared with both MDD and EBD participants, who showed similar scores.

Pearson's correlation analysis carried out on EBD participants revealed significant positive associations between depression, anxiety, and negative affect: the more severe 
the depressive symptoms, the higher the anxiety levels $\left(r_{15}=0.67, p=0.004\right)$ and the greater the negative affect $\left(r_{15}=0.58, p=0.018\right)$; the higher the anxiety levels, the greater the negative affect $\left(r_{15}=0.60, p=0.013\right)$. In addition, a negative correlation revealed that the higher the anxiety levels and the lower the positive affect $\left(r_{15}=-0.78\right.$, $p<0.001)$. MDD patients showed the same positive correlations found for EBD participants: the more severe the depressive symptoms, the higher the anxiety levels $\left(r_{23}=0.47, p=0.028\right)$ and the greater the negative affect $\left(r_{23}=0.52, p=0.008\right)$, and the higher the anxiety levels and the greater the negative affect $\left(r_{23}=0.73, p<0.001\right)$. No significant correlations were found in the PDD group.

Considering resting state EEG data, the ANCOVA revealed a significant Group main effect $\left(F_{2,59}=10.10, p<0.001\right)$ and a significant two-way Group $\times$ Site interaction $\left(F_{2,59}=6.04, p<0.01\right)$. Overall, EBD participants had higher beta/alpha ratio than the other two clinical samples (all $p$ s $<0.001$; Figure 2A). In addition, the EBD group exhibited a typical leftward frontal asymmetry, the beta/alpha ratio being greater at F7 than at the F8 site $(p<0.001)$, whereas both MDD and PDD patients showed a bilateral pattern of frontal beta/alpha ratio (Figure 2B).
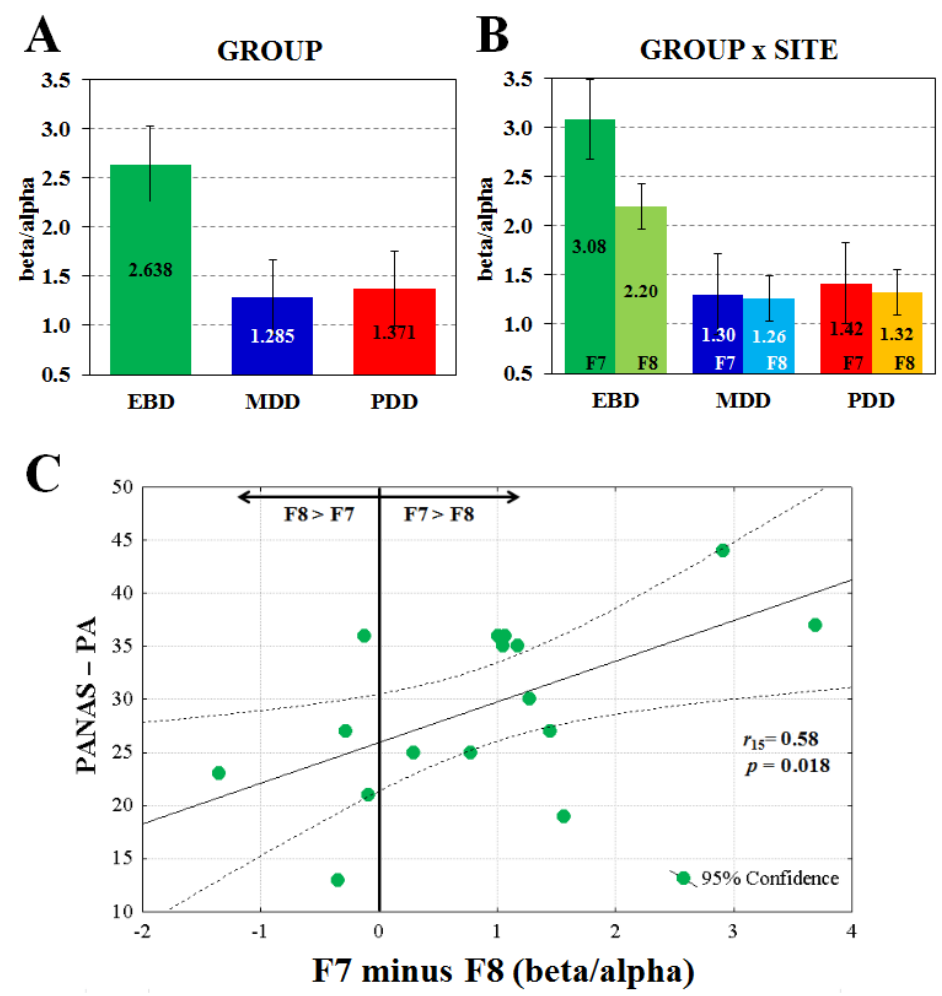

Figure 2. Analysis of resting state frontal beta/alpha ratio. (A) Significant group main effect and (B) significant two-way group $x$ site interaction. (C) Positive Pearson's correlation analysis between EBD participants' frontal laterality activation index and positive affect scores of the PANAS. Asterisks: Significant post hoc comparisons (Newman-Keuls, $p<0.05$ ). Note: EBD, euthymic bipolar disorder (EBD); MDD, major depressive disorder; PDD, persistent depressive disorder.

Pearson's correlation analysis revealed significant positive associations between EBD participants' left frontal beta/alpha ratio and their PANAS-PA scores $\left(r_{15}=0.58, p=0.018\right)$ : the greater the left frontal EEG beta/alpha amplitude (F7), the greater the positive affect (Figure 2C). No correlation was found for MDD and PDD patients.

\section{Discussion}

The present study focused on mood disorder patients characterized by a different severity of symptoms. Together with MDD and PDD patients, EBD outpatients with minimum residual mood symptoms and no current psychotic signs served as a (medicated) 
control group. Making a direct comparison of frontal asymmetry in these three samples in one study allowed us to look for clinical and neurophysiological differences between PDD and MDD. Indeed, so far only limited research has been carried out on PDD, a clinical condition characterized by a poorer outcome due to the persistence of chronic mood symptoms that contribute to worsening the patients' quality of life [2]. For this reason, in PDD patients we expected a pattern of more severe clinical symptoms and more altered/inverted frontal asymmetry as compared with MDD. We used EEG relative beta over alpha band ratio as a measure of frontal activation, a method that allows to normalize spectral energy across participants (see Section 4.3 for details).

Results confirmed that EBD can be reasonably considered a good control group, characterized by no depressive or anxiety symptoms, with high positive and low negative affect (PANAS), and a typical leftward greater frontal activation at rest comparable to that found on healthy controls [33]. These results are in line with past research on both frontal asymmetry in stabilized bipolar patients without symptoms [6], and healthy individuals free from psychiatric disorders used as control group [9,12-14,33]. In addition, EBD outpatients exhibited a positive correlation between a left frontal laterality activation index and positive affect. In line with our hypothesis, the greater the activation at left frontal site, the greater the positive affect.

Electrophysiological results on the two depressed samples showed a different pattern of results. First, both MDD and PDD patients revealed overall frontal inhibition with respect to the EBD control group. This finding is in agreement with consistent past literature pointing to a dysfunction of the frontal cortex in clinically depressed patients [6-11]. This hypofrontality may therefore represent a core landmark of most severe psychiatric disorders, given that schizophrenia (SZ) patients also exhibit hypofrontality, especially at left prefrontal sites [34,35].

As a further important result, compared to EBD patients, both depressed groups exhibited a bilateral pattern of decreased beta amplitude, thus revealing similar symmetrical frontal hypoactivation. This is in line with current literature showing a lack of leftward frontal activity in depressed patients $[9,12-14,16-18])$. In a healthy brain, the functional hierarchy between and within hemispheres points to the dominance of the left prefrontal cortex over the right one, and over the left posterior regions, for many cognitive, metacognitive, and high-level functions. According to Crow [36,37], this left/right and anterior/posterior asymmetry (represented by this human-specific brain torque) is at the origin of Homo sapiens speciation and consciousness, but alterations of this asymmetry would lead to unfavorable psychiatric disorders, such as SZ [34,35,38], as well as to the most severe forms of bipolar and depressive disorders [13]. Interestingly, the electrodes here analyzed $(F 7, F 8)$ were placed over the left and right inferior frontal gyrus, more precisely over the pars orbitalis [39] including Broca's area and the underlying insula [40]. A large number of neuroimaging studies have demonstrated the leading role of the left prefrontal cortex not only on most sub-domains of language (Broca's area [41,42]), but also on attention, working memory, emotion regulation, planning and decision making $[43,44]$. Thus, the reduced dominant activation of the left prefrontal cortex over the right homologous might well explain the neurophysiological altered substrate and related psychiatric symptoms common to type one bipolar disorder, mood disorders and schizophrenia spectrum disorders, including mood oscillations, delusions, though disorders, lack of self-awareness and emotional control, as well as a number of related cognitive impairments (e.g., working memory, attention, decision making, language, planning, etc.).

Moreover, we expected to find a difference between PDD and MDD as, at clinical level, dysthymic patients are affected by more severe symptoms, but at a neurophysiological level the two groups showed a similar pattern of results. MDD and PDD patients showed no correlations between frontal activity and positive/negative affect. Concerning the severity of clinical symptoms, after adjusting for education levels, the analyses of symptom severity revealed a linear gradient on depressive mood signs assessed with the HAM-D scale [31], as EBD outpatients showed, on average, normal or clinically remitting scores (i.e., 
0-7 range), MDD patients showed mild severity scores (i.e., 8-13 range) and PDD patients reported mild to moderate severity scores (i.e., 14-17 range). Furthermore, PDD patients exhibited the highest levels of anxiety [32], the lowest positive affect (PANAS-PA [29]) and the highest negative affect (PANAS-NA [29]) compared with both EBD and MDD patients. This pattern of results support Klein and Santiago's [4] suggestions that, even though the extent of mood symptoms is overall less severe compared with an acute episode in MDD patients [3], the long-lasting persistence of a depressed mood for almost every day for long periods [1] actually depicts a chronic condition marked by important effects on patients quality of life.

In summary, the two domains here analyzed, the clinical and the neurophysiological one, appeared relatively independent as the analysis of clinical symptoms distinguished PDD from MDD, whereas frontal EEG asymmetry showed clear differences between EBD and the two depressed groups, but not between these two latter groups. This points to the existence of a similar neurophysiological substrate (and vulnerability) in the two depressed groups. On the other hand, the clinical signs might be more influenced by contextual and environmental variables related to personal exposition to stressful family-related and mood-evoking events. It is possible that the more pessimistic attitude and psychological style (e.g., greater trait anxiety) of PDD patients leads to a behavior triggering negative feedback from the surrounding environment and this, in turn, would reinforce patients' negative perception of personal events. Therefore, a difference between the PDD and MDD groups can be devised by considering the two domains here analyzed together: a common altered neurophysiological substrate represented by hypofrontality plus a lack of leftward frontal asymmetry, and a different clinical pattern on the main scales of anxiety, depressive symptoms and state affect.

Future directions of this experimental theme could test the above-mentioned interpretation in additional experiments in which frontal asymmetry is measured during specific tasks and/or stimuli prompting negative affect and bias in PDD and MDD patients. We can expect the first group to be more sensitive and more responsive to negative-emotion eliciting stimuli.

In addition, it is possible that the tool used here based on quantitative EEG (qEEG) is not sensitive enough to detect the subtle differences that exist between MDD and PDD. Frontal asymmetry, although frequently used as a neurophysiological marker of depressive symptoms, is only one among all the possible markers of cortical alteration in psychiatric disorders. Cortical connectivity could be another interesting index possibly evidencing greater alterations, represented by excessive connectivity among frontal and other areas in PDD at the expense of other regions and processes underlying a normal and more flexible response to the environment. In line with this, it is worthwhile to mention a recent study showing, in individuals selected for high traits of worry, strong functional connectivity between Broca's area and the ipsilateral amygdala which is activated by negative stimuli. Thus, worrying tends to hyperactivate this amygdala-prefrontal cortex loop, strengthening negative thoughts and interpretations that represent one of the main clinical features of anxious-depressed patients: ruminations [45]. Given the high levels of anxiety measured in PDD patients, a similar experimental design could be applied to the study of connectivity in this neglected sample of patients.

Limitations of the present study are represented by the comparison of psychiatric samples only, a choice with the advantage of controlling for chronic administration of medical treatments, but with a limit in the lack of a direct comparison of psychiatric samples with healthy individuals (nevertheless, we mentioned our past study on healthy individuals). Also, additional tests aimed at measuring cognitive alterations due to the chronic evolution of mental disorders together with the effects of pharmacological treatments would allow a more comprehensive characterization and matching of the clinical disorders. 


\section{Materials and Methods}

\subsection{Participants}

This study has been approved by the Ethics Committee of the Department of General Psychology (Padova, Italy), and adheres to the principles of the Declaration of Helsinki.

Sixty-three native Italian adults ( 44 females, mean age 55 years, $\mathrm{SD} \pm 11$ years, range 29-74 years) suffering from mood disorders gave their written informed consent to take part in the experiment (Table 1). All patients were fully right-handed, on average $94.92 \%$ (SD $\pm 7.90 \%$, range $75-100 \%$ ), according to the Edinburgh Handedness Inventory [30]. Following DSM-IV-TR criteria [46], considering an acute phase of the disorder which had to last for more than 6 months, 25 individuals were diagnosed as major depressive disorder (MDD) patients from the Psychiatric Unit of Mood Disorders-Psychiatric Clinic, Neuroscience Department (University of Padova, Padova, Italy) and from the Psychiatric Unit of the Orlandi Hospital in Bussolengo, Italy (ULSS 9). 21 outpatients at the Villa Santa Chiara clinic in Verona, Italy, and from the Psychiatric Unit of the Orlandi Hospital in Bussolengo, Italy (ULSS 9), fulfilled the criteria of persistent depressive disorder (PDD). All these patients had been diagnosed with PDD (ongoing or lifetime), with or without major depressive episodes both concurrent with the disorder or not, and did not receive a diagnosis of bipolar disorder nor of a disorder within the schizophrenia spectrum. The remaining 17 participants were recruited at the Mood Disorders Outpatient Unit of the Padova University Hospital and they received a diagnosis of bipolar disorder (type I or II) for at least one year: at the moment of the experiment's data collection, they were non-remitting outpatients and they were in a euthymic state (Young Mania Rating Scale (YMRS; [47]) scores being lower than 8). All euthymic bipolar disorder (EBD) patients showed minimum residual mood symptoms with no current psychotic symptoms, satisfying a (sub)clinical condition referred to as euthymia. For this reason, they served as a control group.

All patients were undergoing treatment with various types of drugs at the time the data were collected (Table 2) and were diagnosed by psychiatrists at their respective facility, either with the Mini-International Neuropsychiatric Interview [48] or with the Structured Clinical Interview for DSM Disorders [49]. Prior to the experimental session, patients also filled out the Hamilton Rating Scale for Depression (HAM-D; [31]), the State-Trait Anxiety Inventory $-Y$ in the state form (STAI-Y1; [32]) and the Positive and Negative Affect Schedule (PANAS; [29]).

\subsection{Apparatus and Physiological Recordings}

EEG data were continuously recorded in direct current (DC) mode with a low-pass filter set to $100 \mathrm{~Hz}$, sampling rate of $500 \mathrm{~Hz}$, and amplitude resolution of $0.168 \mu \mathrm{V} / \mathrm{bin}$. Impedance was kept below $5 \mathrm{~K} \Omega$. EEG was measured by means of 38 tin electrodes, using SynAmps amplifiers (NeuroScan Labs, Sterling, VA, USA), 31 mounted (i.e., Fp1, FPz, Fp2, F3, Fz, F4, FC3, FCz, FC4, C3, Cz, C4, CP3, CPz, CP4, P3, Pz, P4, F7, F8, FT7, FT8, T7, T8, TP7, TP8, P7, P8, O1, Oz, O2) on an elastic cap (ElectroCap: Eaton, OH, USA) according to the International 10-20 system [40]; the other seven electrodes were applied below each eye (Io1, Io2), on the two external canthii (F9, F10), on the Nasion (Nz), and on the mastoids (M1, M2). Cz was used as reference during the EEG recording.

\subsection{Experimental Procedure and Data Analysis}

Preliminary, socio-demographical data (Table 1) were analyzed with one-way analysis of variance (ANOVA), using the Newman-Keuls test $(p<0.05)$ for post hoc comparisons, whereas gender distributions were analyzed with separate between-group chi-squared tests. As these analyses revealed higher education levels in EBD than in both MDD and PDD groups, we included the education variable as a covariate in the analysis of the severity of mood symptoms, assessed with the HAM-D, STAI-Y1 and PANAS scales. Post hoc analysis of covariance (ANCOVA) comparisons were computed using the Newman- 
Keuls test $(p<0.05)$. In addition, Pearson's correlations were carried out for each group to investigate the associations among the clinical scales we collected.

Table 2. Pharmacological treatment of the three patient groups.

\begin{tabular}{|c|c|c|c|c|}
\hline & & $\operatorname{EBD}(n=17)$ & $\operatorname{MMD}(n=25)$ & $\operatorname{PDD}(n=21)$ \\
\hline \multirow[t]{7}{*}{ Mood Stabilizers } & & 14 & 8 & 2 \\
\hline & Carbolithium & 4 & 0 & 1 \\
\hline & Antiepileptics & 10 & 8 & 1 \\
\hline & Valproate & 7 & 2 & 1 \\
\hline & Lamotrigine & 2 & 5 & \\
\hline & Gabapentin & 1 & & \\
\hline & Pregabalin & & 1 & \\
\hline \multirow[t]{11}{*}{ Antipsychotics } & & 16 & 5 & 15 \\
\hline & Atypical & 16 & 5 & 7 \\
\hline & Quetiapine & 10 & 2 & 3 \\
\hline & Asenapine & 4 & & \\
\hline & Aripiprazole & 2 & 2 & 1 \\
\hline & Olanzapine & & & 3 \\
\hline & Risperidone & & 1 & \\
\hline & Typical & 0 & 0 & 8 \\
\hline & Perphenazine & & & 5 \\
\hline & Phenothiazine & & & 1 \\
\hline & $\begin{array}{l}\text { Promazine } \\
\text { hydrochloride }\end{array}$ & & & 2 \\
\hline \multirow[t]{22}{*}{ Antidepressant } & & 5 & 31 & 29 \\
\hline & NDRI (Norepinephrine-Dopamine Reuptake Inhibitors) & 0 & 0 & 2 \\
\hline & Bupropion & & & 2 \\
\hline & SARI (Serotonin Antagonist and Reuptake Inhibitors) & 0 & 1 & 5 \\
\hline & Trazodone & & 1 & 2 \\
\hline & Vortioxetine & & & 3 \\
\hline & SNRI (Serotonin-Norepinephrine Reuptake Inhibitors) & 2 & 11 & 7 \\
\hline & Duloxetine & & 4 & 1 \\
\hline & Venlafaxine & & 5 & 4 \\
\hline & Mirtazapine & 2 & 2 & 2 \\
\hline & SSRI (Selective Serotonin Reuptake Inhibitors) & 3 & 15 & 11 \\
\hline & Citalopram & & 3 & \\
\hline & Escitalopram & 1 & 4 & 2 \\
\hline & Fluoxetine & & 3 & 1 \\
\hline & Fluvoxamine & 1 & & \\
\hline & Paroxetine & & 2 & 5 \\
\hline & Sertraline & 1 & 3 & 3 \\
\hline & Tricyclics & 0 & 4 & 4 \\
\hline & Amitriptyline & & 1 & 2 \\
\hline & Clomipramine & & 2 & \\
\hline & Dosulepin & & & 2 \\
\hline & Nortriptyline & & 1 & \\
\hline
\end{tabular}


Table 2. Cont.

\begin{tabular}{|c|c|c|c|c|c|}
\hline & & & $\operatorname{EBD}(n=17)$ & MMD $(n=25)$ & $\operatorname{PDD}(n=21)$ \\
\hline \multirow[t]{12}{*}{ Anxiolytics } & & & 1 & 8 & 31 \\
\hline & Benzodiazepine & & 1 & 8 & 30 \\
\hline & & Alprazolam & & 1 & 7 \\
\hline & & Bromazepam & & & 1 \\
\hline & & Clonazepam & & 3 & 5 \\
\hline & & Delorazepam & & 1 & 2 \\
\hline & & Diazepam & 1 & & \\
\hline & & Flurazepam & & 1 & 7 \\
\hline & & Lorazepam & & 2 & 4 \\
\hline & & Triazolam & & & 4 \\
\hline & Thienodiazepine & & 0 & 0 & 1 \\
\hline & & Etiazolam & & & 1 \\
\hline
\end{tabular}

Note: EBD, euthymic bipolar disorder; MDD, major depressive disorder; PDD, persistent depressive disorder.

The electrophysiological session consisted of a 5-min, open-eyes resting state EEG recording, asking participants to sit relaxed, with their eyes open and focused on a fixation cross placed in the center of a computer screen. EEG was continuously recorded and stored for analysis. Data were off-line re-referenced to the average reference. After blinking and eye movement artifacts correction, following the eye movement modeling approach of Ille and colleagues [50] using the BESA software (Brain Electrical Source Analysis, 5.1 version), all EEG data were divided into 150 2048-ms time intervals: indeed, given the constraint of BESA to use $2^{n}$ samples, we needed to force the width of each interval to 1024 samples, corresponding to a 2048-ms interval with $0.488 \mathrm{~Hz}$ FFT resolution. An artifact rejection procedure was performed during each interval, with both amplitude and derivative thresholds (with respect to time) $(250 \mu \mathrm{V}$ and $100 \mu \mathrm{V} / \mathrm{ms}$, respectively). The remaining epochs were also visually inspected to remove any residual artifacts: on average, $88.60 \%$ of the epochs were accepted, equally distributed among groups. After windowing each interval with a tapered cosine, the FFT was averaged across those epochs that were finally free of residual artifacts.

In a past experiment co-measuring PET and EEG in a healthy sample at rest, greater global and voxel-wise positive correlation was found between high frequency EEG bands (namely high-beta and gamma) and brain metabolism, whereas alpha EEG band showed the greatest negative correlation [26]. The combination of alpha and beta EEG bands (beta/alpha ratio) adopted in the present study allows to maximize the most activated cortical regions and, at the same time, normalizes the spectral activation across subjects (as the ratio indicates a percentage of beta over alpha amplitude [51]) so that individuals with greater spectral energy do not weigh more statistically than those with smaller energy.

Thus, for statistical purposes, we calculated an activation index [51] as the ratio between high-beta (in the text indicated for simplicity as beta only; 20-35 Hz, effective $\beta$ range $=20.50-35.14 \mathrm{~Hz}$ ) and alpha bands $(8-12 \mathrm{~Hz}$, effective $\alpha$ range $=8.30-11.71 \mathrm{~Hz})$ on F7 and F8 electrodes. Therefore, the statistical analysis included the group factor (three levels: EBD vs. MDD vs. PDD) and the site factor (two levels: F7 vs. F8). In addition, we included Education as a covariate, as preliminary socio-demographical analyses revealed higher education levels in EBD than both MDD and PDD groups. Post hoc analysis of COVAriance (ANCOVA) comparisons were computed using the Newman-Keuls test $(p<0.05)$.

Finally, to ascertain whether patients' frontal asymmetry represented a physiological correlate that was significantly associated with the severity of clinical symptoms, Pearson's correlation analyses were carried out between F7 minus F8 laterality indices and the scores obtained at the HAM-D, STAI-Y1, and PANAS subscales (PA and NA). Laterality indices 
were positive when participants' activation index was higher in F7 (left hemisphere) and negative when they had greater activation in F8 (right hemisphere).

Author Contributions: Conceptualization, investigation, methodology, validation and project administration: C.S., M.B. and A.A. Formal analysis, writing—original draft preparation and funding acquisition: C.S. Data curation, supervision and writing—review and editing: F.F., M.B. and A.A. All authors have read and agreed to the published version of the manuscript.

Funding: This study was supported by a grant, PRIN 2017, from the Italian Ministry of Education and Research, project n. 20178NNRCR_003 to C.S. The present work was also carried out within the scope of the research program Dipartimenti di Eccellenza (art.1, commi 314-337 legge 232/2016), which was supported by a grant from MIUR to the Department of General Psychology, University of Padua.

Institutional Review Board Statement: The study was conducted according to the guidelines of the Declaration of Helsinki, and approved by the Ethics Committee of Psychology Area, University of Padova (protocol code 1240, date of approval 9 January 2013) and the Ethics Committee of Azienda ULSS 9 Scaligera. U.O.C. Assistenza Farmaceutica Territoriale (protocol code 101356, date of approval 18 June 2019).

Informed Consent Statement: Not applicable.

Data Availability Statement: The data that support the findings of this study are available on request from the corresponding author. The data are not publicly available, due to privacy or ethical restrictions.

Conflicts of Interest: The authors declare no conflict of interest.

$\begin{array}{ll}\text { Abbreviations } \\ \text { EBD } & \text { Euthymic Bipolar Disorder } \\ \text { MDD } & \text { Major Depressive Disorder } \\ \text { PDD } & \text { Persistent Depressive Disorder (Dysthymia) } \\ \text { EEG } & \text { Electroencephalography } \\ \text { HAM-D } & \text { Hamilton Rating Scale for Depression } \\ \text { PANAS-PA } & \text { Positive and Negative Affect Schedule-Positive Affect } \\ \text { PANAS-NA } & \text { Positive and Negative Affect Schedule-Negative Affect } \\ \text { STAI-Y1 } & \text { State-Trait Anxiety Inventory-state form }\end{array}$

\section{References}

1. American Psychiatric Association. Diagnostic and Statistical Manual of Mental Disorder, 5th ed.; DSM-5; American Psychiatric Association: Arlington, TX, USA, 2013.

2. Klein, D.N.; Schwartz, J.E.; Rose, S.; Leader, J.B. Five-year course and outcome of dysthymic disorder: A prospective, naturalistic follow-up study. Am. J. Psychiatry 2000, 157, 931-939. [CrossRef] [PubMed]

3. Yang, T.; Dunner, D.L. Differential subtyping of depression. Depress. Anxiety 2001, 13, 11-17. [CrossRef]

4. Klein, D.N.; Santiago, N.J. Dysthymia and chronic depression: Introduction, classification, risk factors, and course. J. Clin. Psychol. 2003, 59, 807-816. [CrossRef] [PubMed]

5. Schramm, E.; Klein, D.N.; Elsaesser, M.; Furukawa, T.A.; Domschke, K. Review of dysthymia and persistent depressive disorder: History, correlates, and clinical implications. Lancet Psychiatry 2020, 7, 801-812. [CrossRef]

6. Ravindran, A.V.; Smith, A.; Cameron, C.; Bhatla, R.; Cameron, I.; Georgescu, T.M.; Hogan, M.J. Toward a functional neuroanatomy of dysthymia: A functional magnetic resonance imaging study. J. Affect. Disord. 2009, 119, 9-15. [CrossRef]

7. Vilgis, V.; Chen, J.; Silk, T.J.; Cunnington, R.; Vance, A. Frontoparietal function in young people with dysthymic disorder (DSM-5: Persistent depressive disorder) during spatial working memory. J. Affect. Disord. 2014, 160, 34-42. [CrossRef]

8. Stewart, J.L.; Coan, J.A.; Towers, D.N.; Allen, J.J. Frontal EEG asymmetry during emotional challenge differentiates individuals with and without lifetime major depressive disorder. J. Affect. Disord. 2011, 129, 167-174. [CrossRef]

9. Allen, J.J.B.; Urry, H.L.; Hitt, S.K.; Coan, J.A. The stability of resting frontal electroencephalographic asymmetry in depression. Psychophysiology 2004, 41, 269-280. [CrossRef]

10. Coan, J.A.; Allen, J.J.B. The state and trait nature of frontal EEG asymmetry in emotion. In The Asymmetrical Brain, 2nd ed.; Hugdahl, K., Davidson, R.J., Eds.; MIT Press: Cambridge, MA, USA, 2003; pp. 565-615.

11. Davidson, R.J. Anterior electrophysiological asymmetries, emotion, and depression: Conceptual and methodological conundrums. Psychophysiology 1998, 35, 607-614. [CrossRef]

12. Palmiero, M.; Piccardi, L. Frontal EEG asymmetry of mood: A mini-review. Front. Behav. Neurosci. 2017, 11, 224. [CrossRef] 
13. Spironelli, C.; Maffei, A.; Romeo, Z.; Piazzon, G.; Padovan, G.; Magnolfi, G.; Pasini, I.; Homen, F.G.; Concari, G.; Angrilli, A. Evidence of language-related left hypofrontality in Major Depression: An EEG Beta band study. Sci. Rep. 2020, $10,8166$. [CrossRef]

14. Van der Vinne, N.; Vollebregt, M.A.; van Putten, M.J.A.M.; Arns, M. Frontal alpha asymmetry as a diagnostic marker in depression: Fact or fiction? A meta-analysis. NeuroImage Clin. 2017, 16, 79-87. [CrossRef]

15. Nusslock, R.; Walden, K.; Harmon-Jones, E. Asymmetrical frontal cortical activity associated with differential risk for mood and anxiety disorder symptoms: An RDoC perspective. Int. J. Psychophysiol. 2015, 98, 249-261. [CrossRef]

16. Allen, J.J.B.; Iacono, W.G.; Depue, R.A.; Arbisi, P. Regional electroencephalographic asymmetries in bipolar seasonal affective disorder before and after exposure to bright light. Biol. Psychiatry 1993, 33, 642-646. [CrossRef]

17. Allen, J.J.B.; Coan, J.A.; Nazarian, M. What's the difference? Issue and assumptions in the use of difference scores and other metrics of anterior brain asymmetry in emotion. Biol. Psychol. 2004, 67, 183-218. [CrossRef]

18. Stewart, J.L.; Coan, J.A.; Towers, D.N.; Allen, J.J.B. Resting and task-elicited prefrontal EEG alpha asymmetry in depression: Support for the capability model. Psychophysiology 2014, 51, 446-455. [CrossRef]

19. Pizzagalli, D.A.; Nitschke, J.B.; Oakes, T.R.; Hendrick, A.M.; Horras, K.A.; Larson, C.L.; Abercrombie, H.C.; Schaefer, S.M.; Koger, J.V.; Benca, R.M.; et al. Brain electrical tomography in depression: The importance of symptom severity, anxiety, and melancholic feature. Biol. Psychiatry 2002, 52, 73-85. [CrossRef]

20. Reid, S.A.; Duke, L.M.; Allen, J.J.B. Resting frontal electroencephalographic asymmetry in depression: Inconsistencies suggest the need to identify mediating factors. Psychophysiology 1998, 35, 389-404. [CrossRef]

21. Jesulola, E.; Christopher, F.; Sharpleya, C.F.; Bitsikab, V.; Agnewa, L.L.; Wilson, P. Frontal alpha asymmetry as a pathway to behavioural withdrawal in depression: Research findings and issues. Behav. Brain Res. 2015, 292, 56-67. [CrossRef]

22. Koek, R.; Yerevanian, B.; Tachiki, K.; Smith, J.; Alcock, J.; Kopelowicz, K. Hemispheric asymmetry in depression and mania. A longitudinal QEEG study in bipolar disorder. J. Affect. Disord. 1999, 53, 109-122. [CrossRef]

23. Diykh, M.; Li, Y.; Wen, P.; Li, T. Complex networks approach for depth of anesthesia assessment. Measurement 2018, 119, 178-198. [CrossRef]

24. Diykh, M.; Abdulla, S.; Saleh, K.; Deo, R.C. Fractal dimension undirected correlation graph-based support vector machine model for identification of focal and non-focal electroencephalography signals. Biomed. Signal Process. Control 2019, $54,101611$. [CrossRef]

25. Murakami, M.; Kominami, D.; Leibnitz, K.; Murata, M. Drawing Inspiration from Human Brain Networks: Construction of Interconnected Virtual Networks. Sensors 2018, 18, 1133. [CrossRef]

26. Oakes, T.R.; Pizzagalli, D.A.; Hendrick, A.M.; Horras, K.A.; Larson, C.L.; Abercrombie, H.C.; Schaefer, S.M.; Koger, J.V.; Davidson, R.J. Functional Coupling of Simultaneous Electrical and Metabolic Activity in the Human Brain. Hum. Brain Mapp. 2004, 21, 257-270. [CrossRef]

27. Moncrieff, J.; Cohen, D. Do antidepressants cure or create abnormal brain states? PLoS Med. 2006, 3, e240. [CrossRef]

28. Fornaro, M.; Anastasia, A.; Novello, S.; Fusco, A.; Pariano, R.; De Berardis, D.; Solmi, M.; Veronese, N.; Stubbs, B.; Vieta, E.; et al. The emergence of loss of efficacy during antidepressant drug treatment for major depressive disorder: An integrative review of evidence, mechanisms, and clinical implications. Pharmacol. Res. 2019, 139, 494-502. [CrossRef]

29. Watson, D.C.; Lee, A.; Tellegen, A. Development and validation of brief measures of positive and negative affect: The PANAS scales. J. Personal. Soc. Psychol. 1988, 54, 1063-1070. [CrossRef]

30. Oldfield, R.C. The assessment and analysis of handedness: The Edinburgh inventory. Neuropsychologia 1971, 9, 97-113. [CrossRef]

31. Hamilton, M. A rating scale for depression. J. Neurol. Neurosurg. Psychiatry 1960, 23, 56. [CrossRef]

32. Spielberger, C.D.; Gorsuch, R.L.; Lushene, R.; Vagg, P.R.; Jacobs, G.A. Manual for the State-Trait Anxiety Inventory (Form Y); Psychologist Press: Palo Alto, CA, USA, 1983.

33. Spironelli, C.; Angrilli, A. Posture used in fMRI-PET elicits reduced cortical activity and altered hemispheric asymmetry with respect to sitting position: An EEG resting state study. Front. Hum. Neurosci. 2017, 11, 621. [CrossRef]

34. Angrilli, A.; Spironelli, C.; Elbert, T.; Crow, T.J.; Marano, G.; Stegagno, L. Schizophrenia as failure of left hemispheric dominance for the phonological component of language. PLoS ONE 2009, 4, e4507. [CrossRef] [PubMed]

35. Spironelli, C.; Angrilli, A.; Calogero, A.; Stegagno, L. Delta EEG band as a marker of left hypofrontality in schizophrenia patients. Schizophr. Bull. 2011, 37, 757-767. [CrossRef] [PubMed]

36. Crow, T.J. Schizophrenia as failure of hemispheric dominance for language. Trends Neurosci. 1997, $20,33-343$.

37. Crow, T.J. Schizophrenia as the price that Homo sapiens pays for language: A resolution of the central paradox in the origin of the species. Brain Res. Rev. 2000, 31, 118-129. [CrossRef]

38. Spironelli, C.; Angrilli, A.; Stegagno, L. Failure of language lateralization in schizophrenia patients: An ERP study on early linguistic components. J. Psychiatry Neurosci. 2008, 33, 235-243.

39. Cutini, S.; Scatturin, P.; Zorzi, M. A new method based on ICBM152 head surface for probe placement in multichannel fNIRS. NeuroIamge 2011, 54, 919-927. [CrossRef]

40. Oostenveld, R.; Praamstra, P. The five percent electrode system for high-resolution EEG and ERP measurements. Clin. Neurophysiol. 2001, 112, 713-719. [CrossRef]

41. Bookheimer, S. Functional MRI of language: New approaches to understanding the cortical organization of semantic processing. Annu. Rev. Neurosci. 2002, 25, 151-188. [CrossRef] 
42. Hagoort, P. On Broca, brain, and binding: A new framework. Trends Cogn. Sci. 2005, 9, 416-423. [CrossRef]

43. Fink, G.R.; Manjaly, Z.M.; Stephan, K.E.; Gurd, J.M.; Zilles, K.; Amunts, K.; Marshall, J.C. A Role for Broca's Area beyond Language Processing: Evidence from Neuropsychology and fMRI. In Broca's Region; Godzinsky, Y., Amunts, K., Eds.; Oxford University Press: New York, NY, USA, 2006; pp. 254-268.

44. Brunyé, T.T. Non-invasive Brain Stimulation Effects on the Perceptual and Cognitive Processes Underlying Decision-making: A Mini Review. J. Cogn. Enhanc. 2021, 5, 233-244. [CrossRef]

45. Guha, A.; Spielberg, J.M.; Lake, J.; Popov, T.; Heller, W.; Yee, C.M.; Miller, G.A. Effective Connectivity between Broca's Area and Amygdala as a Mechanism of Top-Down Control in Worry. Clin. Psychol. Sci. 2020, 8, 84-98. [CrossRef]

46. American Psychiatric Association. Diagnostic and Statistical Manual of Mental Disorders, 4th ed.; Text Revised; American Psychiatric Association: Washington, DC, USA, 2000.

47. Young, R.C.; Biggs, J.T.; Ziegler, V.E.; Meyer, D.A. A rating scale for mania: Reliability, validity and sensitivity. Br. J. Psychiatry 1978, 133, 429-435. [CrossRef]

48. Sheehan, D.V.; Lecrubier, Y.; Sheehan, K.H.; Amorim, P.; Janavs, J.; Weiller, E.; Hergueta, T.; Baker, R.; Dunbar, G.C. The Mini-International Neuropsychiatric Interview (M.I.N.I.): The development and validation of a structured diagnostic psychiatric interview for DSM-IV and ICD-10. J. Clin. Psychiatry 1998, 59, 22-33.

49. First, M.B.; Spitzer, R.L.; Williams, J.B.W.; Gibbon, M. Structured clinical interview for DSM-IV Axis I disorders (SCID-I). In Handbook of Psychiatric Measures; Rush, A.J., Jr., First, M.B., Blacker, D., Eds.; American Psychiatric Association: Washington, DC, USA, 2000; pp. 49-53.

50. Ille, N.; Berg, P.; Scherg, M. Artifact correction of the ongoing EEG using spatial filters based on artifact and brain signal topographies. J. Clin. Neurophysiol. 2002, 19, 113-124. [CrossRef]

51. Spironelli, C.; Borella, E. Working memory training and cortical arousal in healthy older adults: A resting state EEG pilot study. Front. Aging Neurosci. 2021, 13, 718965. [CrossRef] 